\title{
Role of ultrasonography in evaluation of tendons abnormalities in hand and fingers
}

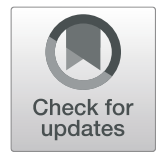

\author{
Ahmed Mohammed Fekry El-Deek and Elsayed Mohamed Abd El-Hamid Hassan Dawood
}

\begin{abstract}
Background: Characterization of tendon pathologies of the hand and fingers remains problematic, despite advances in imaging. By using clinical history and imaging appearance, one can determine the diagnosis. Ultrasonography (USG) may be used as the first imaging modality when MRI unavailable or has a higher cost for patients.

Purpose: Assessment of role of USG in evaluation of tendons abnormalities in hand and fingers

Results: USG detected 20 of 20 cases (100\%) of tenosynovitis, 8 of 10 cases (80\%) of trigger finger, 10 of 12 cases (83.3\%) of tendon tear, 3 of 3 cases (100\%) of foreign body impaction, 12 of 12 cases (100\%) of simple ganglion, and 3 of 3 cases (100\%) of solid masses with sensitivity, specificity, and accuracy 93.8\%, 97.8\%, and 95.8\%, respectively.

Conclusion: USG is a powerful, easy, and inexpensive imaging tool that allows accurate assessment of the hand and finger's tendons abnormalities.
\end{abstract}

Keywords: Hand pain, Magnetic resonance imaging, Tendons abnormalities, Ultrasonography

\section{Background}

Tendons abnormalities of the hand and the fingers are common disorders, particularly among athletes and in the elderly. They may be symptomatic degenerative changes, inflammatory, or rupture. Tendon disorders are a common cause of pain and loss of function. Chronic tendon disorders are much more common than acute injuries and are the result of overuse or age-related tendon degeneration [1]. The quality of ultrasonographic (USG) assessment of anatomical structures in the hand has highly improved over the last time. The development of high resolution with the highest possible frequency of modern ultrasound equipment and the superficial location of most tendons allows the spectrum of tendon abnormalities to be easily depicted with USG [2,3]. The role of USG in the assessment of tendon disorders is steadily increasing due to its low cost, fast, wide-spread availability, and noninvasively together with the possibility it offers a dynamic assessment of the flexor and extensor tendons, collateral ligaments, and supporting structures of the finger, such as

\footnotetext{
* Correspondence: Sayeddawood71@yahoo.com

Diagnostic Radiology Department, Faculty of medicine, Al-Azhar University, New Damietta, Egypt
}

\section{Methods}

This study was conducted during the period between March 2016 and September 2016 at the Radiodiagnosis Department. It included 60 patients with age ranging from 16 to 55 years (mean $\pm \mathrm{SD}=32.0 \pm 13$ ) complaining from pain or masses of the hands and the fingers, referred for assessment of tendons causes of such pain and swellings. There were 35 male $(58.3 \%)$ and 25 female (41.7\%) patients. Patients with a history of operative intervention were excluded.

The study was approved by the scientific and ethical committee of the hospital. Written and verbal consents were obtained from all patients.

Patients were subjected to full clinical history including the patient's name, age, sex, complaint (hand and finger pain or swelling), and associated symptoms. Clinical examination was made by the referring physician. 
Patients underwent USG followed by MRI conducted by two different radiologists, and both were blinded to the imaging findings of the other modality to reduce bias. Imaging findings on both modalities were then statistically correlated.

\section{Technique}

\section{Ultrasound examinations}

USG examinations were performed with TOSHIBA (Xario 200) using superficial linear transducer 7.5 MHZ. During superficial USG examination of the hand and fingers, the patient was examined while sitting upright, with the hand placed on a cushion.

For examination of the anatomical snuff box, the hand was placed in prone position. Proximally, the snuff box is demarcated by the radial styloid, and distally by the base of the thumb metacarpal. Its radial boundary is formed by two tendons (extensor pollicis brevis and abductor pollicis longus) and on the ulnar aspect by extensor pollicis longus. The floor of the snuff box is formed by the scaphoid proximally and the trapezium distally. It contains the radial artery and cephalic vein.

For examination of palmar spaces, the patient puts the hand in supinated position. We move the transducer axially from proximal to distal to examine the lateral, medial, and central spaces. The palm is divided into three spaces by two septa passing from the palmar aponeurosis to the thumb and little finger metacarpals. The lateral space contains thenar muscles, the medial contains hypothenar muscles, and the central contains long flexor tendons, lumbricals, the superficial and deep palmar arches, and median nerve. For the examination of the flexor tendons of the palm of the hand, namely the flexor digitorum superficialis and flexor digitorum profundus, we move the transducer distal to carpal tunnel to the level of metacarpal region. Axial and longitudinal plane images should be obtained over this tendon (Fig. 1a).

For the examination of the flexor tendons of the fingers, namely the flexor digitorum superficialis and flexor digitorum profundus, we move the transducer to the fingers. Axial and longitudinal plane images should be obtained over this tendon (Fig. 1b, c).

For the examination of the extensor tendons of the hand and fingers, namely the extensor digitorum, we move to the dorsal aspect of the hand and fingers. Axial and longitudinal plane images should be obtained over this tendon (Fig. 1d-f).

\section{MRI examinations}

All patients were examined by conventional MRI. The MRI machine used was PHILIPS (Achiva). Using dedicated wrist coil the examination protocol included coronal, sagittal, and axial planes. Coronal and sagittal fast spin-echo T1-weighted (TR/TE, 400/15), coronal and axial fast spin-echo T2-weighted (TR/TE, 2000/60), coronal gradient (TR/TE, 450/20), and coronal STIR (TR/ TE, 2000/65) sequences with a field of view of $12 \mathrm{~cm}$

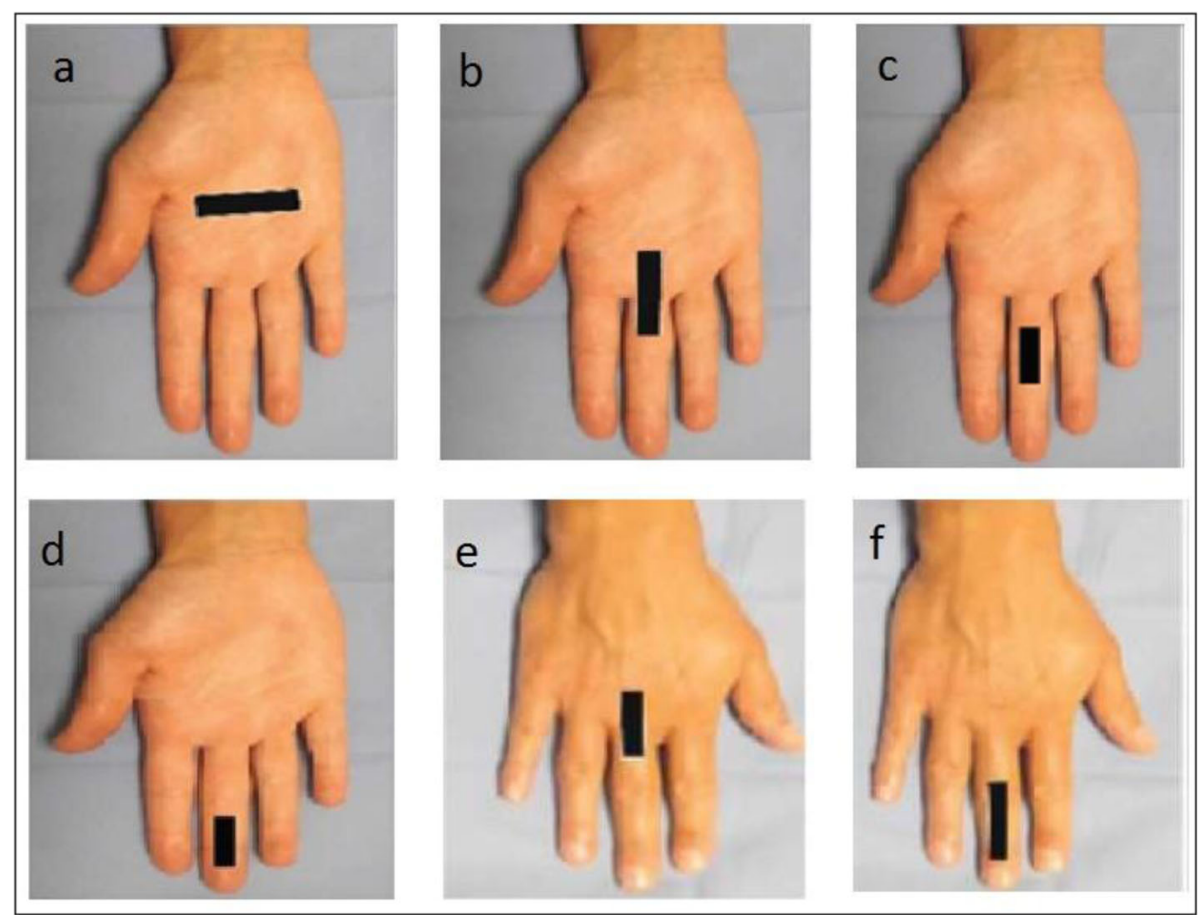

Fig. 1 Hand positions during USG examination of flexor and extensor tendons of the hand and fingers 
were used. Slice thickness was $2.5 \mathrm{~mm}$ with $2 \mathrm{~mm}$ interslice gap.

\section{Statistical analysis}

Data were statistically described in terms of mean \pm standard deviation $( \pm \mathrm{SD})$, and range, or frequencies (number of cases) and percentages when appropriate. All statistical calculations were done using computer program SPSS (Statistical Package for the Social Science;
SPSS Inc., Chicago, IL, USA) Release 15 for Microsoft Windows (2006).

\section{Results}

This study included 60 patients, 35 males (58.3\%) and 25 females $(41.7 \%)$ with an age range from 16 to 55 years (mean \pm SD, $32 \pm 13.0$ ). They presented with different pathologies, tenosynovitis (20 cases), trigger finger (10 cases), tendon tear (12 cases), simple ganglion (12 cases), solid masses ( 3 cases), and foreign body ( 3 cases). This

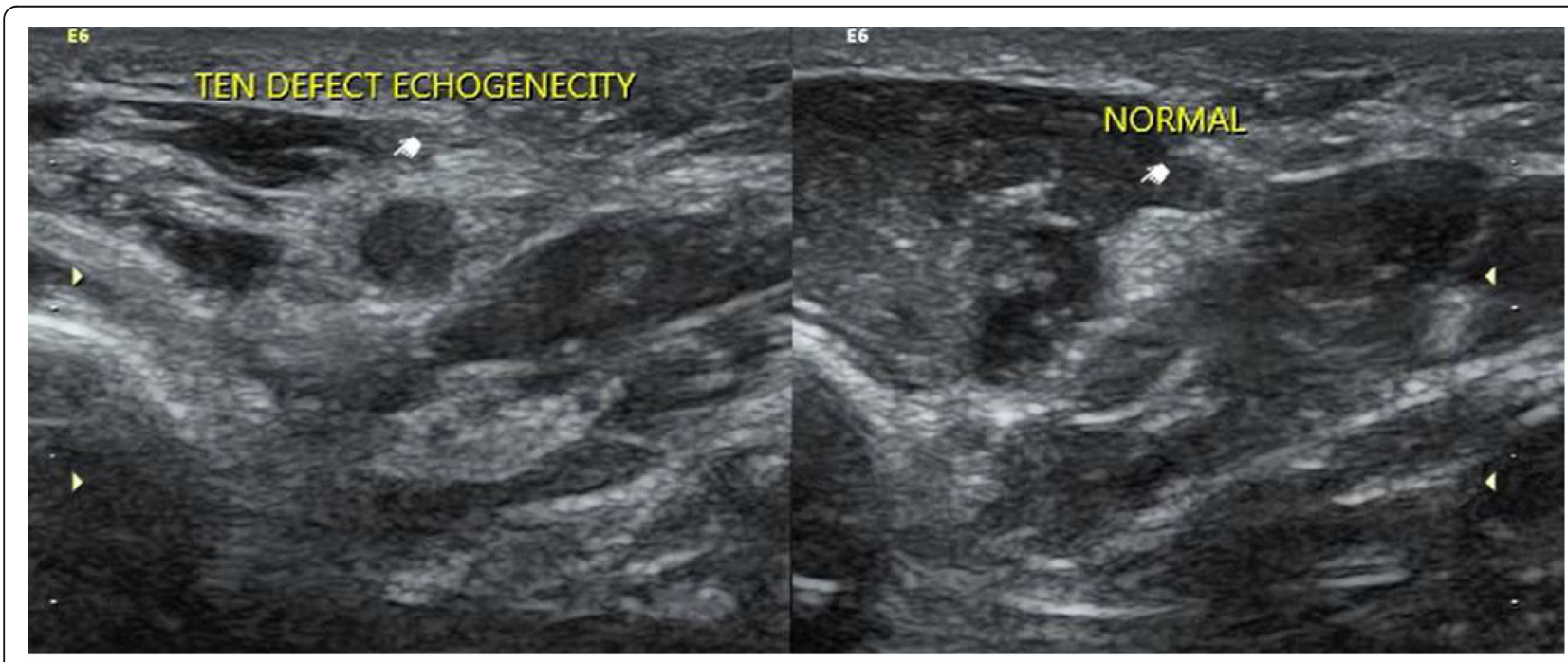

\section{Axial view}

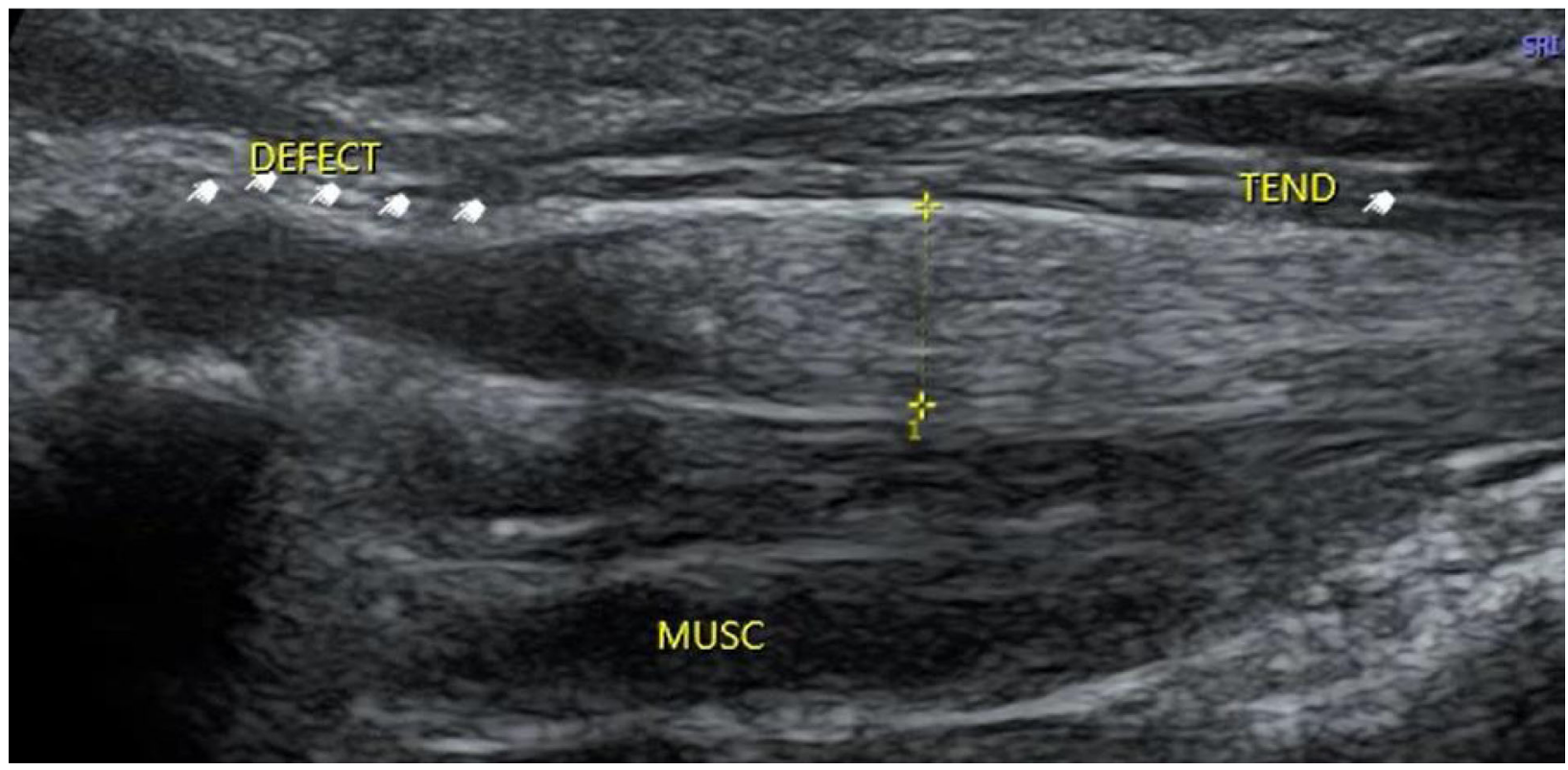

\section{Longitudinal view}

Fig. 2 Female patient 32 years with a history of cut wound at thenar area from 3 months. Comment: there is thickened tendon with hypoechoic area replacing its normal course ... complete tendon tear 
diagnosis was confirmed by MRI examination and postoperative follow-up if possible (Figs. 2, 3, and 4).

USG detected 20 from $20(100 \%)$ cases with tenosynovitis. It detected 8 from $10(80 \%)$ cases of trigger finger and unable to diagnose 2 cases $(20 \%)$ as normal study. It detected 10 from 12 (83.3\%) cases with complete tendon tear and misdiagnosed 2 cases $(16.7 \%)$ as a tenosynovitis which were diagnosed by MRI as partial tendon tear. USG detected 12 of $12(100 \%)$ cases with simple ganglions and confirmed by post-operative follow-up. It detected 3 from $3(100 \%)$ cases with solid lesion and 3 from $3(100 \%)$ cases with foreign body. Over all, USG correctly diagnosed 56 cases (true positive), falsely diagnosed 2 cases as a normal study (false negative) which were diagnosed as trigger finger by MRI. Two false positive cases were diagnosed by USG; they had been diagnosed as tenosynovitis which were diagnosed by MRI as partial tendon tear surrounded by an inflammatory reaction (Table 1 ).

Sensitivity, specificity, and accuracy were calculated for USG assessment of tendon abnormalities of the hand and fingers; they were $93.8 \%, 97.8 \%$, and $95.8 \%$, respectively (Table 2).

\section{Discussion}

USG technology has been rapidly advancing over the past few years. The development of high-resolution transducers has increased the potential of ultrasound to evaluate superficial anatomical structures of the hand and fingers [2]. In view of reliability and a good sensitivity:specificity ratio, USG has been proposed as the gold standard in assessing tendon involvement [6] (Tables 3 and 4).

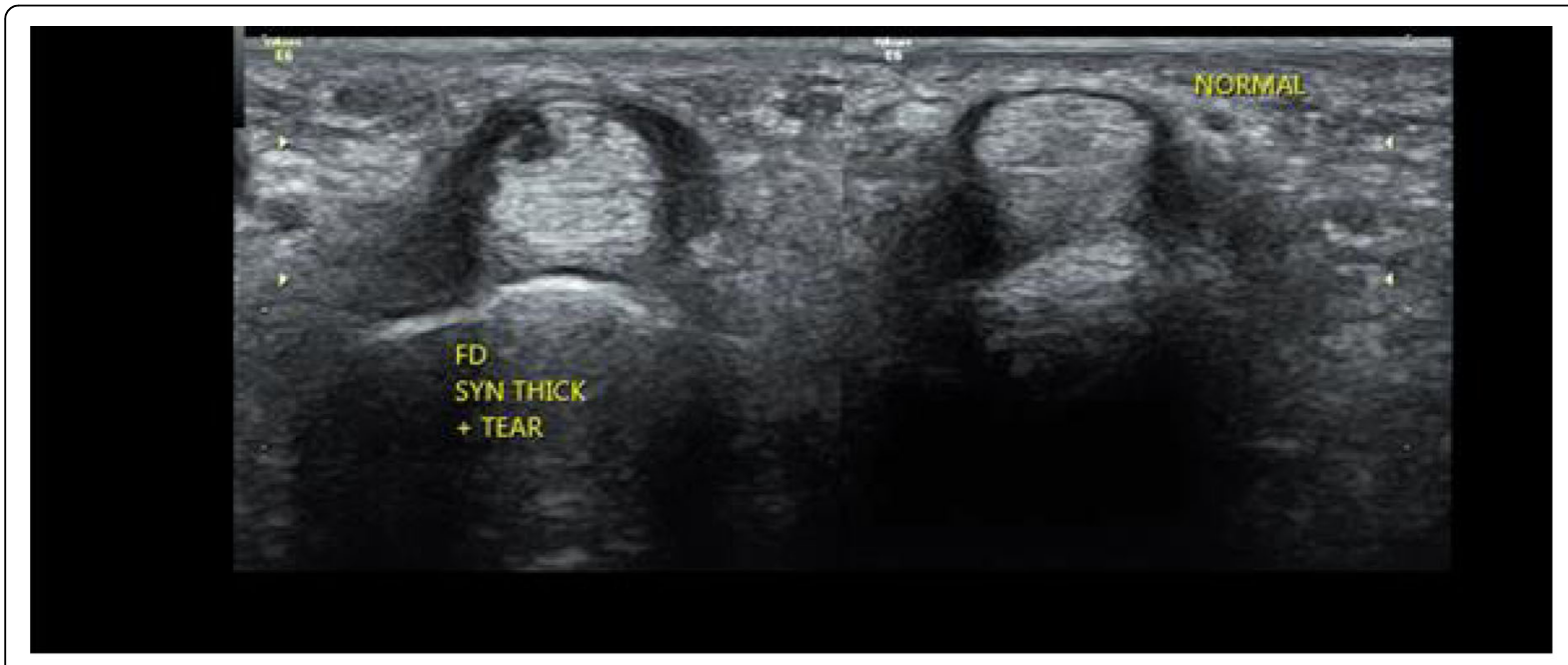

Axial view

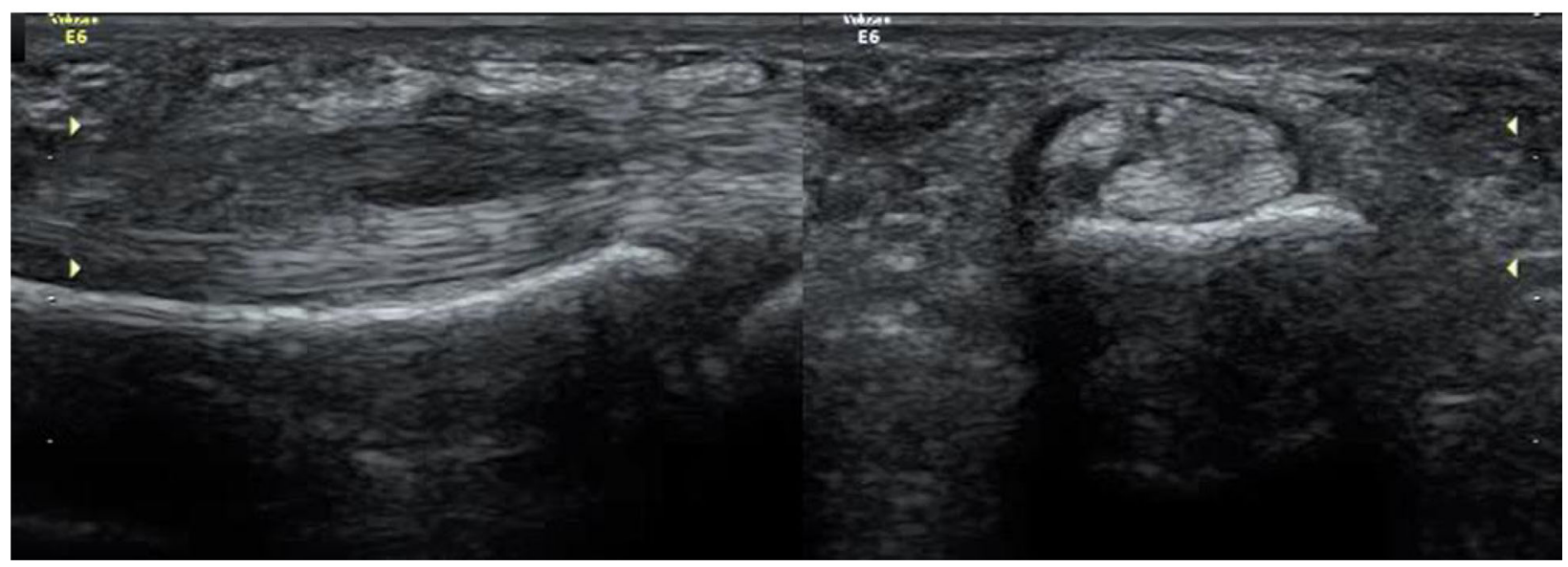

\section{Axial and longitudinal}

Fig. 3 Female patient 45 years presented with pain at ring finger with movement. Comment: there is intra substance hypo echoic area seen in flexor tendon of ring finger at proximal phalanx associated with thickened synovium partial tendon tear 


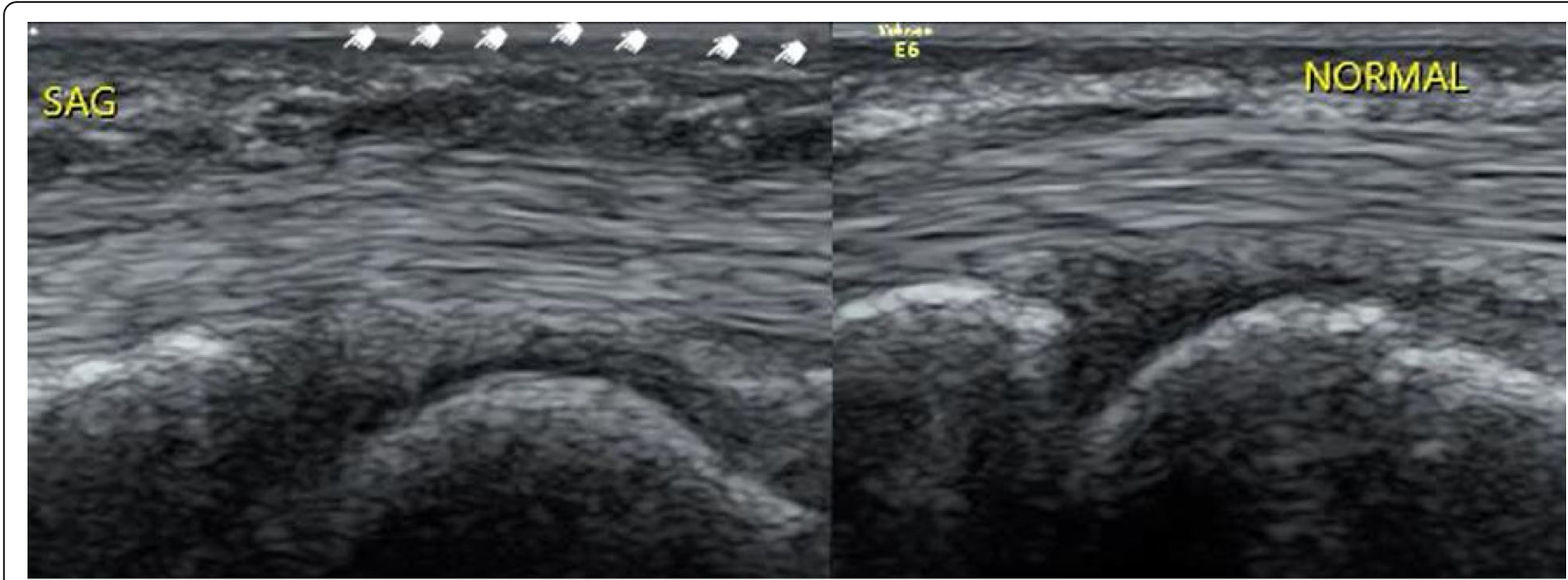

\section{Sagittal}

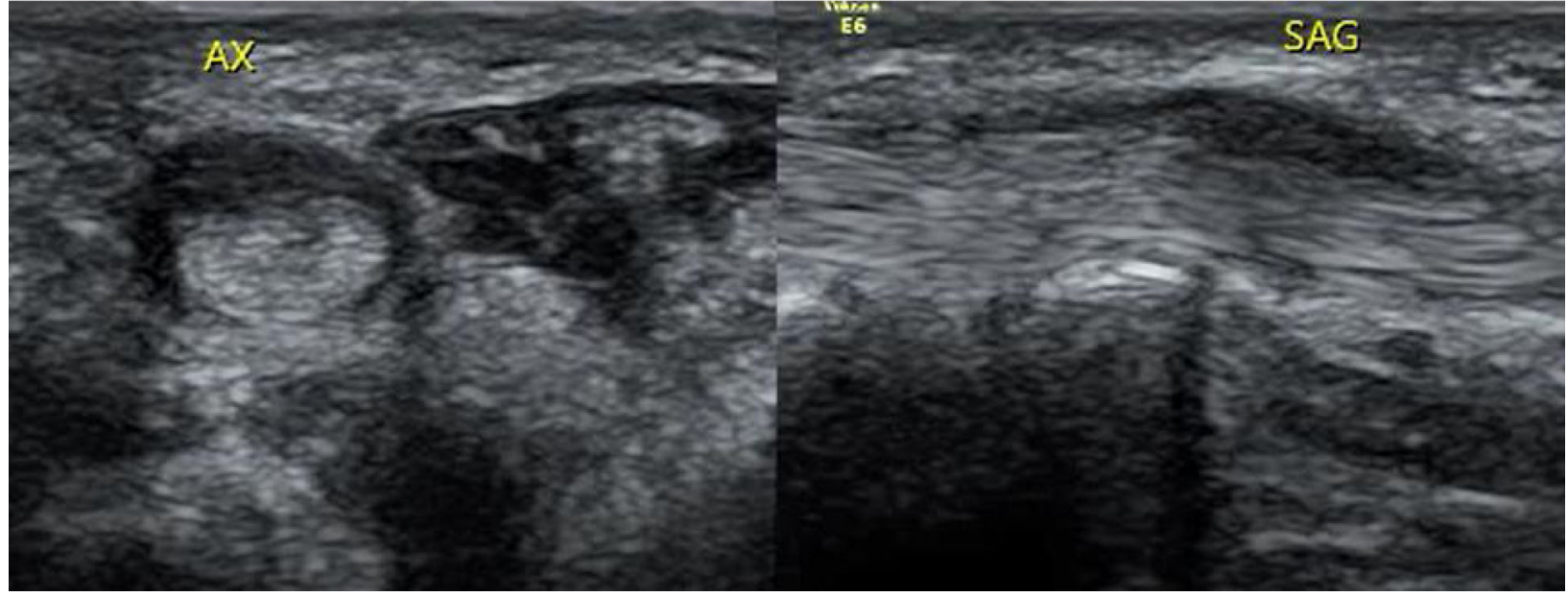

\section{Axial and sagittal}

Fig. 4 Male patient 52 years presented by clinical trigger finger with history of minor trauma. Comment: there is focal thickened hypoechoic synovial sheath compressing the flexor tendon of ring finger at distal metacarpal bone with intact tendon ... focal synovial thickening

Table 1 Correlation between USG and MRI findings for tendon pathologies of hand and fingers

\begin{tabular}{lllllll}
\hline & \multicolumn{2}{l}{ MRI } & & & USG \\
\cline { 2 - 3 } & No. & $\%$ & & No. & $\%$ \\
\hline Tenosynovitis & 20 & 33.3 & & 20 & 33.3 \\
Trigger finger & 10 & 16.7 & & 8 & 13.3 \\
Tendon tear & 12 & 20 & & 10 & 16.7 \\
Simple ganglion & 12 & 20 & & 12 & 20 \\
Solid mass & 3 & 5 & & 3 & 5 \\
Foreign body & 3 & 5 & & 3 & 5 \\
Undiagnosed or misdiagnosed & 0 & 0 & & 4 & 6.7 \\
\hline
\end{tabular}

In the current study, USG diagnosed 20 of 20 cases $(100 \%)$ of isolated tenosynovitis with peritendinous fluid collection and increased vascularity. USG is highly sensitive and specific in the diagnosis of tenosynovitis. The first evidence of the USG's ability to reveal tendon pathology was by Grassi et al. [7] detecting signs of finger flexor and/or extensor tenosynovitis in 18 (80\%) of 20 patients.

Table 2 Overall sensitivity, specificity and accuracy of MRI and USG results

\begin{tabular}{llll}
\hline & Sensitivity & Specificity & Accuracy \\
\hline MRI & $100 \%$ & $100 \%$ & $100 \%$ \\
US & $93.3 \%$ & $97.8 \%$ & $95.8 \%$ \\
\hline
\end{tabular}


Table 3 Comparison between RT and LT side affection

\begin{tabular}{lll}
\hline & No. of cases & $\%$ \\
\hline RT hand & 40 & 66.7 \\
LT hand & 20 & 33.3 \\
Both hands & 0 & 0 \\
\hline
\end{tabular}

In our study, USG diagnosed trigger finger associated with tendinosis and tenosynovitis in 8 of 10 cases $(80 \%)$ by detecting thickening and hypervascularity of pulley system. Our findings were in agreement with the study done by Vuillemin et al. [8] in that the sonographic signs of stenosing tenosynovitis of the digits have also been well described. Hypoechoic thickening of pulley A1 next to the metacarpo-phalangeal joint is a constant finding which may be associated with hypervascularization on Doppler imaging (91\% of all cases), peritendinous effusion, tendinosis, or tendinosis with effusion.

Among studied cases, USG diagnosed 10 of 12 cases (83.3\%) of tendon tear. Discontinuity of the tendons with hematoma in the acute cases was the finding. Hodgson et al. [9] had published that US is powerful for assessment of the tendons with high sensitivity and specificity and over all accuracy in detection of tendon tears.

In our study, USG detected foreign bodies in 3 of 3 cases $(100 \%)$ with extension to tendon fibers with surrounding fluid collection, this is in agreement with Tahmasebi et al. [10] who reported a higher accuracy and sensitivity (90.2\% and $97.9 \%$, respectively), for USG in detecting radiolucent foreign bodies. If highresolution USG is available, we recommend it as the first imaging modality for evaluating the patients with clinically suspicious radiolucent foreign body because of its availability, high sensitivity, and absence of radiation. In patients with a history of soft tissue foreign body and negative radiography, we recommend USG as the most important diagnostic tool before discharging patients. USG gives important information about the size, depth, and relationship of foreign bodies to other structures such as vessels and tendons and makes exploration easier for the surgeon. Furthermore, an important advantage of USG is the possibility of real-time guided removal of foreign body under sterile condition, and due to its safety and less complication rate, it may replace surgical exploration.

In our study, USG diagnosed 12 of 12 cases (100\%) of simple ganglions related to tendon sheathes and 3 of 3 (100\%) cases of solid lesions related to tendon sheaths

Table 4 Comparison between affected tendon groups

\begin{tabular}{lll}
\hline & No. of cases & $\%$ \\
\hline Flexor tendon groups & 32 & 53.3 \\
Extensor tendon groups & 28 & 46.7 \\
\hline
\end{tabular}

with intact fibers of the tendon and diagnosed by histopathological study as giant cell tumor of tendon sheath. Our study is in agreement with study done by Faruch et al. [11] in that USG is a good technique to assess a mass of the hand. USG allows identifying the anatomical structures from which the lesions originate. USG is essential to analyze the tumor matrix, by identifying if the lesion is cystic or solid. A cyst appears as a well-circumscribed walled lesion, anechoic with posterior acoustic enhancement. Color Doppler ultrasonography is essential to assess the vascularity of the soft tissue mass. The vascularity of masses is a criterion of aggressiveness. Finally, ultrasonography is also part of the treatment plan by guiding infiltration or biopsy procedures.

In our study, we had found that sensitivity, specificity, and accuracy of USG assessment of tendons abnormalities of the hand $93.8 \%, 97.8 \%$, and $95.8 \%$, respectively. This is in agreement with the study done by Robinson [12] who reported that US is an efficient and accurate imaging method for evaluation of common tendon abnormalities.

\section{Conclusion}

USG of hands should be a fundamental part of the imaging protocol when tendons abnormalities of the hands and the fingers (tenosynovitis, trigger finger, tendon tear, tendon related masses, and foreign body) are suspected, as it achieves the highest level of diagnostic confidence as it is powerful, easy, and inexpensive imaging tool.

\section{Abbreviation \\ USG: Ultrasonography}

\section{Acknowledgements}

The authors thank all the study participants for their patience and support.

\section{Authors' contributions}

AM discussed the idea of the work, reviewed literature, was responsible for interpreting and reporting MRI cases, planned and designed the work, interpreted the data, acquired the data and saved the data, performed statistical analysis, prepared figures and tables, drafted the manuscript, and revised and edited the manuscript. EM suggested the idea of the work, reviewed literature, was responsible for operating, interpreted and reported musculoskeletal ultrasound cases, planned and designed the work, interpreted the data, acquired the data and saved the data, shared in statistical analysis, prepared figures and tables, drafted the manuscript, and revised and edited the manuscript. All authors have read and approved the manuscript.

\section{Funding}

No funding was received.

\section{Availability of data and materials}

All data and material included in our study are available. The data sets used and analyzed during the current study are available on reasonable request.

\section{Ethics approval and consent to participate}

This study was approved by the Research Ethics Committee of the Faculty of Medicine at Al-Azhar University (New Damietta), in Egypt, on 23February 2016, reference number of approval: ADIMIRB 131/16. Informed consent obtained from study participants was written and assigned by participants or their first-degree relatives. 


\section{Consent for publication}

All authors have agreed to the submission to the journal.

\section{Competing interests}

The authors declare that they have no competing interests.

Received: 2 December 2019 Accepted: 5 December 2019

Published online: 30 December 2019

\section{References}

1. Nissman DB, Dahiya N (2014) Ultrasonography of tendons. Musculoskeletal Imaging Section, Department of Radiology, University of North Carolina School of Medicine

2. Furrer M, Schweizer A, Rufibach K, Meuli-Simmen C (2009) The value of ultrasonography in hand surgery. Am Assoc Hand Surg 4:385-390

3. Bodor M, Fullerton B (2010) Ultrasonography of the hand, wrist, and elbow. Department of Neurological Surgery, UCSF Medical Center, and Physical Medicine and Rehabilitation, Sports and Electrodiagnostic Medicine, pp 509-531

4. Bianchi S, Martinoli C (2007) Ultrasound of the musculoskeletal system, 1st edn. Springer, Heidelberg, pp 134-146

5. Lee PT, Hans LB, Beltran J et al (2008) Imaging of the musculoskeletal system, volume I. Elsevier, Philadelphia, pp 399-413

6. Patil P, Dasgupta B (2012) Role of diagnostic ultrasound in the assessment of musculoskeletal diseases. Ther Adv Musculoskel Dis 4(5):341-355

7. Grassi W, Tittarelli E, Blasetti P, Pirani O, Cervini C (1995) Finger tendon involvement in rheumatoid arthritis. Evaluation with high-frequency sonography. Arthritis Rheum 38:786-794

8. Vuillemin V, Guerini H, Bard H, Morvan G (2012) Stenosing tenosynovitis. J Ultrasound 15:20e28

9. Hodgson RJ, O'connor PJ, Grainger AJ (2012) Tendon and ligament imaging. Br J Radiol 85:1157-1172

10. Tahmasebi M, Zareizadeh H, Motamedfar A (2014) Accuracy of ultrasonography in detecting radiolucent soft-tissue foreign bodies. Indian J Radiol Imaging 24(2):196-200

11. Faruch MB, Lapègue F, Brun C et al (2015) Tumors and pseudotumors of the hand: the role of imaging. Diagnostic and Interventional Imaging https://doi.org/10.1016/j.diii.2015.09.011

12. Robinson P (2009) Sonography of common tendon injuries. American Roentgen Ray Society AJR 193:607-618

\section{Publisher's Note}

Springer Nature remains neutral with regard to jurisdictional claims in published maps and institutional affiliations.

\section{Submit your manuscript to a SpringerOpen ${ }^{\odot}$ journal and benefit from:}

- Convenient online submission

- Rigorous peer review

- Open access: articles freely available online

- High visibility within the field

- Retaining the copyright to your article 